\title{
A prostate cancer patient with isolated lung metastases: a case report
}

\author{
Li-Xin Wu ${ }^{1 \#}$, Lei Lei ${ }^{2 \#}$, You-Cai Zhu ${ }^{1}$, Kai-Qi Du ${ }^{1}$, Xiao-Feng Li $^{1}$, Hua-Fei Chen ${ }^{1}$, Wen-Xian Wang ${ }^{2}$, \\ Chun-Wei $\mathrm{Xu}^{3}$
}

${ }^{1}$ Department of Thoracic Disease Center, Zhejiang Rongjun Hospital, The Third Affiliated Hospital of Jiaxing University, Jiaxing 314000 , China; ${ }^{2}$ Department of Chemotherapy, Chinese Academy of Sciences University Cancer Hospital (Zhejiang Cancer Hospital), Hangzhou 310022, China; ${ }^{3}$ Department of Respiratory Medicine, Jinling Hospital, Nanjing University School of Medicine, Nanjing 210002, China

"These authors contributed equally to this work.

Correspondence to: Li-Xin Wu, MD, PhD. Department of Thoracic Disease Center, Zhejiang Rongjun Hospital, The Third Affiliated Hospital of Jiaxing University, No. 589 Central West Road, Jiaxing 314000, China. Email: xiaoyaoren101@hotmail.com; Chun-Wei Xu, MD, PhD. Department of Respiratory Medicine, Jinling Hospital, Nanjing University School of Medicine, 305 Zhongshan Road, Nanjing 210002, China. Email: dg1935057@smail.nju.edu.cn.

\begin{abstract}
Pulmonary involvement has been reported in $>40 \%$ of autopsy series in patients with metastatic prostate cancer; however, isolated lung metastases have been documented in $<1 \%$ of cases and $43.5 \%$ (10/23) cases underwent surgical resection and most of them have good outcome. We present a 74-year-old male Gleason high-grade prostate cancer patient with initially negative PSA and isolated pulmonary lesion which was confirmed as lung metastasis by resection. This patient received first-line endocrine therapy with leuprolide and bicalutamide endocrine and had a long-term disease-free follow-up of 3 years. The present patient had isolated lung metastasis with negative PSA, which was very rare in literature. Unexpected longterm disease-free survival was achieved after first-line endocrine therapy in this case with Gleason score of 8 metastatic prostate cancer. Whether or not the path of metastasis in this case was via lymph node jumping (negative lymph node dissection) or hematogenous (usually multiple, in bilateral lungs and lower lung fields) requires further investigation.
\end{abstract}

Keywords: Lung metastasis; prostate cancer; pathology; case report

Submitted Nov 28, 2019. Accepted for publication Jan 06, 2020.

doi: $10.21037 /$ tcr.2020.01.19

View this article at: http://dx.doi.org/10.21037/tcr.2020.01.19

\section{Introduction}

Lung metastases from prostate cancer occur in some terminal patients with multiple metastases; however, isolated lung metastases without concurrent bone or lymph node metastases are extremely rare. It has been reported that isolated lung metastases occur in $<1 \%$ of autopsies involving patients with metastatic prostate cancer (1). In a clinical study of 1,290 patients diagnosed with prostate cancer, 48 had lung metastases and only $11(0.86 \%)$ had solitary nodules (2). Gleason score of 8-10 indicate highgrade prostate cancer and usually associate with unfavorable outcome than lower scores and more aggressive treatments should be considered $(3,4)$. In the current study, we presented an unusual case who was eventually diagnosed as high-grade prostate cancer with the initial discovery of isolated lung metastasis and negative PSA, and harvest longterm disease free survival after first-line endocrine therapy. All protocols in the present study were approved by the Human Clinical and Research Ethics Committees of the Zhejiang Rongjun Hospital (Jiaxing, China). The patient provided written informed consent for the publication of case details and any accompanying images.

\section{Case presentation}

In October 2016, a 74-year-old male non-smoker presented 
A

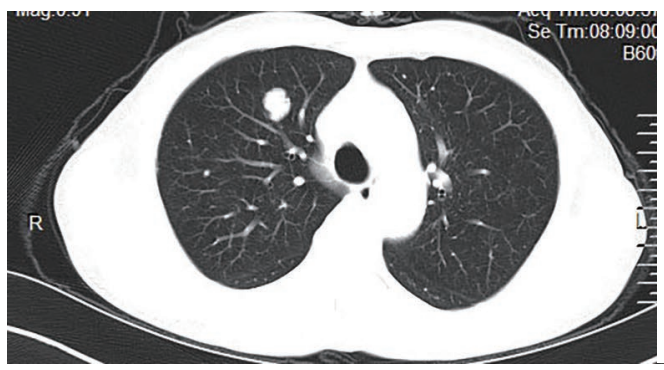

B

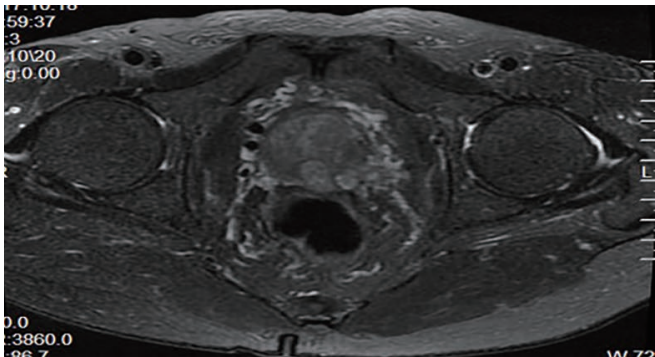

Figure 1 Computed tomography (CT) scans show (A) a mass in right lower lung. (B) Magnetic resonance imaging (MRI) scans show (B) a mass in prostate.

to our hospital for a physical examination, which revealed a pulmonary mass. A computed tomography (CT) scan showed a mass in the right lower lung (Figure 1). The patient had non-special comorbidities, family and psychosocial history. The patient was given treatment options and chose observation. A repeat contrast-enhanced CT one week ago in our hospital showed a nodule in the right upper lobe of the lung with malignant signs, which was considered to be primary lung cancer. Tumor biomarker testing showed the following: alpha fetoprotein (AFP), $2.48 \mathrm{ng} / \mathrm{mL}$; carcinoembryonic antigen (CEA), $2.64 \mathrm{ng} / \mathrm{mL}$; ferritin, $141.0 \mathrm{ng} / \mathrm{mL}$; squamous cell carcinoma-associated antigen, $0.60 \mathrm{ng} / \mathrm{mL}$; carbohydrate antigen, $199<2.00 \mathrm{IU} / \mathrm{mL}$; free PSA, $0.6 \mu \mathrm{g} / \mathrm{L}$; and total PSA, $3.0 \mu \mathrm{g} / \mathrm{L}$. The patient underwent a thoracoscopic radical resection of the right upper lung with electrocautery division of pleural adhesions under general anesthesia. The post-operative pathology findings were as follows: tumor cells: CK5/6 (-); CK7 (-); P63 (-); CK20 (-); TTF-1 (-); Napsin A (+); Ki-67 (+, 30-40\%); Syn (-); CgA (-); CDX-2 (+); PSA (+); and P504S (+) (Figure 2). Then, a next generation sequencing (NGS) analysis of the tumor biopsy identified that the tumor had TP53 p.V216M, NF1 c.3974+1G>A, CD274, CDK6, PIK3CA, SOX2, PREX2 and RICTOR amplification (3D Medicines, Shanghai, China) Based on hematoxylineosin staining and immunohistochemistry, the tumor met the criteria for a poorly differentiated adenocarcinoma (approximately $1.8 \mathrm{~cm}$ in diameter) and was likely metastatic from a primary prostate tumor. A prostate magnetic resonance imaging (MRI) suggested prostatic spaceoccupying lesions involving the seminal vesicles bilaterally (Figure 1), with a high possibility of prostate cancer. Needle aspiration biopsies of the prostate gland were then obtained and the pathologic examination confirmed prostate adenocarcinoma with a Gleason grade score of $4+4=8$ points (prognostic grade group IV). The patient was treated with one subcutaneous injection of leuprolide $(3.75 \mathrm{mg})$ and endocrine therapy [oral bicalutamide $(50 \mathrm{mg} \mathrm{qd})$ ] as firstline therapy until now. During almost 3 years of followup since the first administration of endocrine therapy, every-3-month PSA evaluation and digital examination confirmed the status of disease was stable (Figure 3). This patient tolerated and adherent to the endocrine therapy quite well except grade 1 appetite and pubic hair loss, respectively.

\section{Discussion}

As a common malignancy in elderly males, prostate cancer is one of the major diseases leading to the death of males worldwide. The incidence of prostate cancer ranks first for malignant tumors among males in Western countries and mortality is second only to lung cancer (1). In recent years, the incidence and detection of prostate cancer in China have increased yearly (2). Prostate cancer may metastasize to any organ, including bones, lymph nodes, and lungs. Although lung metastases have been reported in $>40 \%$ of patients with prostate cancer (3-7), cases of isolated lung metastases without concurrent bone or lymph node metastases are uncommon. In the current patient, a $12 \mathrm{~mm} \times 14 \mathrm{~mm}$ round solid nodule in the right upper lobe was detected during physical examination. The mass was an isolated, lobulated lesion with a clear boundary, unevenly enhanced on contrast CT, and had imaging characteristics of a primary lung cancer. Tumor biomarkers, including free and total PSA were normal. Head MRI and ECT bone scans did not reveal metastases. A thoracoscopic radical resection of the right upper lung with electrocautery division of 

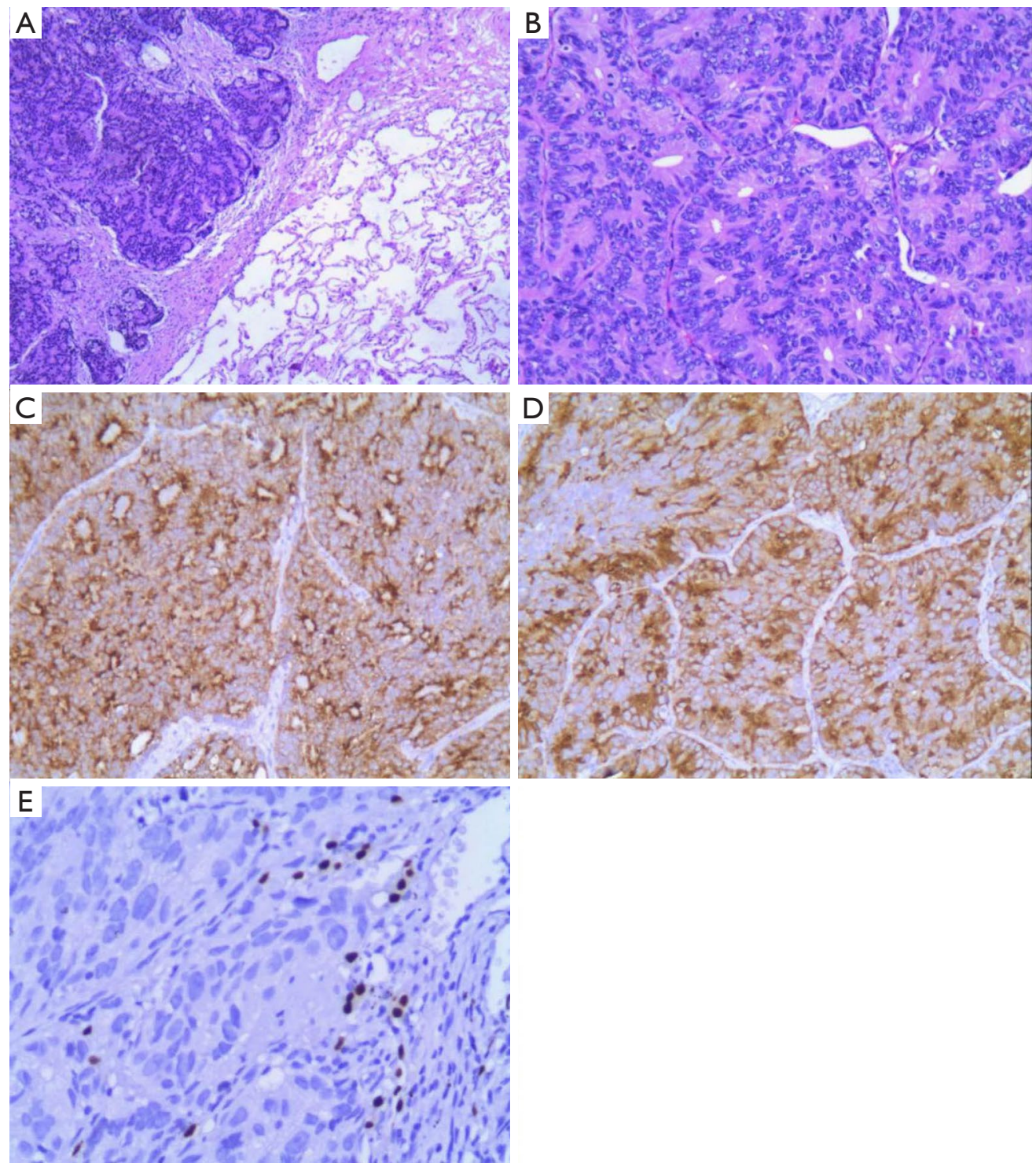

Figure 2 Hematoxylin and eosin staining revealed adenocarcinoma and lung tissue, $(\mathrm{HE} \times 100)(\mathrm{A})$, adenocarcinoma, $(\mathrm{HE} \times 200)(\mathrm{B})$. Immunohistochemical (IHC) analysis revealed that the lung tumor cells were positive for PSA (C) and P504S (D), negative for TTF-1 (E) $(\mathrm{HE} \times 200)$.

pleural adhesions was then performed. The post-operative pathologic examination suggested a poorly differentiated adenocarcinoma (approximately $1.8 \mathrm{~cm}$ in diameter). Immunohistochemical staining was PSA (+), indicating the possibility of prostate adenocarcinoma metastasis. Further examination demonstrated a primary prostate lesion and confirmed the diagnosis of solitary lung metastases from prostate adenocarcinoma.

Prostate cancer is often accompanied by an elevated serum PSA. PSA is a specific marker for the diagnosis of prostate cancer. PSA is normally distributed in the prostate ducts and only has trace amounts in the blood. Prostate cancer can injure duct barriers and lead to the release of PSA into the circulation, thus increasing the level of PSA in the blood. If setting a total PSA $>4 \mu \mathrm{g} / \mathrm{L}$ as a diagnostic cutoff, the sensitivity is $71 \%$ and specificity is only $49 \%$. The current patient had normal serum free and total PSA levels at the time of admission, which reminds us that an increase in the PSA level should not be the only and indispensable standard for early prostate cancer; there is still a possibility 


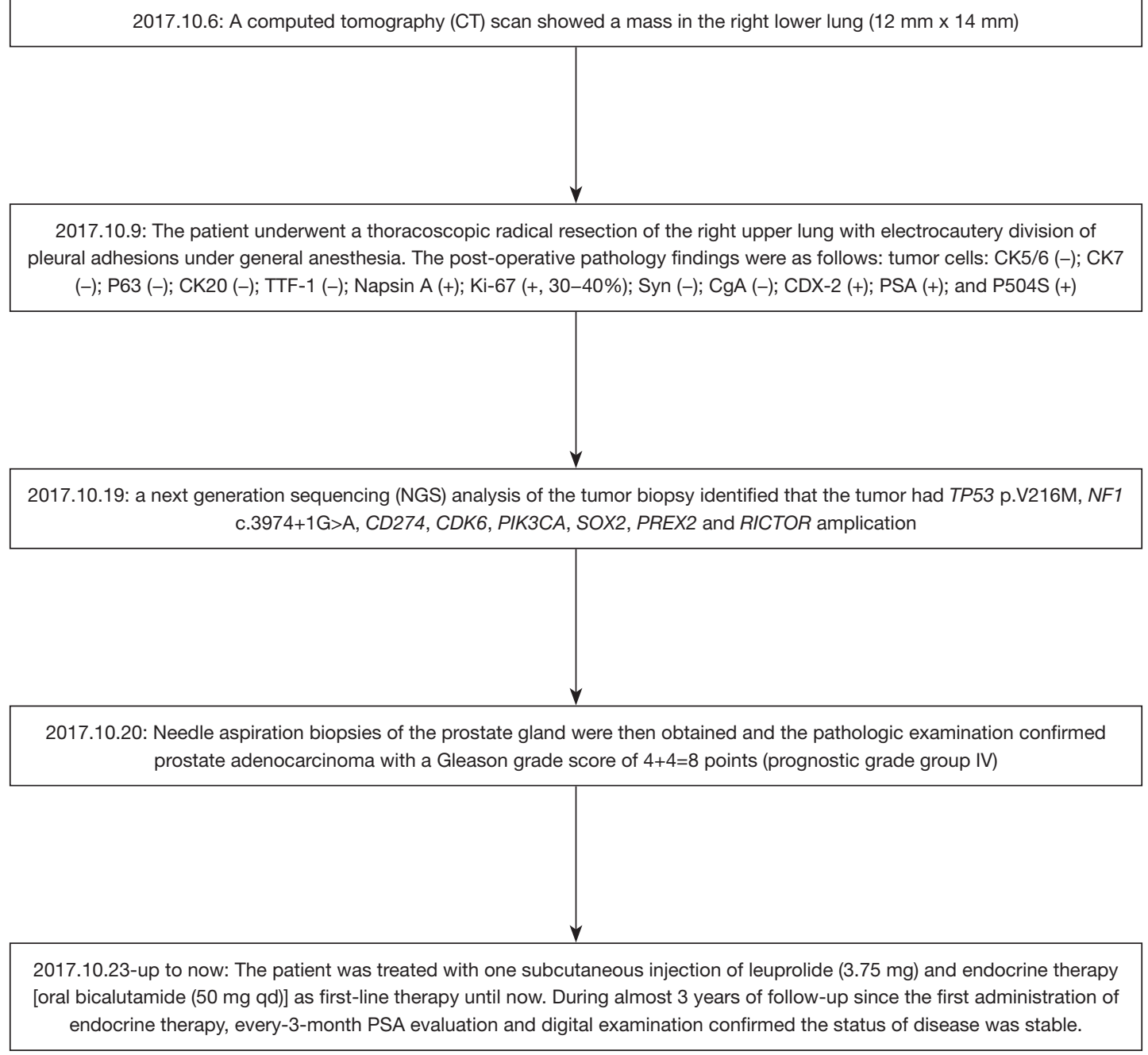

Figure 3 Flow chart of the treatment.

of false negatives $(8,9)$. Prostate cancer generally involves lymph node or bone metastases, with blood metastases less common. Solitary metastases of prostate cancer are defined as the demonstration of metastatic tumors by imaging examination in patients with prostate cancer, metastatic tumors are limited in lymph nodes or bones (non-visceral metastases), and the number of metastases is $\leq 5$ (10). The mechanism of isolated spread to pulmonary metastasis in prostate cancer was unclear.

One limitation in our case report is that the primary lesion in this patient has been evaluated by CT scan instead of standard MRI during follow-up which could have certain impact of results. However, CT scans could be more efficient and economical than MRI especially in long-term follow-up during endocrine therapy.

\section{Take-away lessons}

The present patient had isolated lung metastasis with negative PSA, which was very rare in literature. Unexpected long-term disease-free survival was achieved after firstline endocrine therapy in a Gleason score of 8 metastatic prostate cancer. Whether or not the path of metastasis in this case was via lymph node jumping (negative lymph node dissection) or hematogenous (usually multiple, in bilateral lungs and lower lung fields) requires further investigation.

\section{Acknowledgments}

Funding: Zhejiang Public Welfare Technology Research 
Program (LGJ20H160001), Science and Technology Planning project of Zhejiang Province (LGF19H160002), the Medical Scientific Research Foundation of Zhejiang Province of China (2019RC027), the Technology Bureau of Jiaxing City of Zhejiang Province (2016AY23087, 2017 B Y 18050 , 2018AD 32163 , 2019AD 32097 , 2019AD32266), the Scientific Research Foundation of Zhejiang Medical Association (2018ZYC-B5, 2019ZYC-A76) and Xisike-Hanson Cancer Research Foundation (YHS2019-20).

\section{Footnote}

Conflicts of Interest: All authors have completed the ICMJE uniform disclosure form (available at http://dx.doi. org/10.21037/tcr.2020.01.19). The authors have no conflicts of interest to declare.

Ethical Statement: The authors are accountable for all aspects of the work in ensuring that questions related to the accuracy or integrity of any part of the work are appropriately investigated and resolved. All procedures performed in studies involving human participants were in accordance with the Declaration of Helsinki (as revised in 2013). Written informed consent was obtained from the patient for publication of this manuscript and any accompanying images.

Open Access Statement: This is an Open Access article distributed in accordance with the Creative Commons Attribution-NonCommercial-NoDerivs 4.0 International License (CC BY-NC-ND 4.0), which permits the noncommercial replication and distribution of the article with the strict proviso that no changes or edits are made and the original work is properly cited (including links to both the formal publication through the relevant DOI and the license). See: https://creativecommons.org/licenses/by-nc-nd/4.0/.

\section{References}

1. Saitoh H, Hida M, Shimbo T, et al. Metastatic patterns of prostatic cancer. Correlation between sites and number of organs involved. Cancer 1984;54:3078-84.

2. Fabozzi SJ, Schellhammer PF, el-Mahdi AM. Pulmonary metastases from prostate cancer. Cancer 1995;75:2706-9.

3. Siegel RL, Miller KD, Jemal A. Cancer statistics, 2018. CA Cancer J Clin 2018;68:7-30.

4. Chen W, Zheng R, Baade PD, et al. Cancer statistics in China, 2015. CA Cancer J Clin 2016;66:115-32.

5. Bubendorf L, Schöpfer A, Wagner U, et al. Metastatic patterns of prostate cancer: an autopsy study of 1,589 patients. Hum Pathol 2000;31:578-83.

6. Vinjamoori AH, Jagannathan JP, Shinagare AB, et al. Atypical metastases from prostate cancer: 10-year experience at a single institution. AJR Am J Roentgenol 2012;199:367-72.

7. Muro Y, Kosaka T, Hato T, et al. Coincident detection of lung metastasis of prostate cancer and primary lung cancer: A case report. Can Urol Assoc J 2015;9:E524-6.

8. Rigau M, Ortega I, Mir MC, et al. A three-gene panel on urine increases PSA specificity in the detection of prostate cancer. Prostate 2011;71:1736-45.

9. Cao DL, Ye DW, Zhang HL, et al. A multiplex model of combining gene-based, protein-based, and metabolitebased with positive and negative markers in urine for the early diagnosis of prostate cancer. Prostate 2011;71:700-10.

10. Malik D, Basher RK, Sood A, et al. Herniated Thoracic Spleen Mimicking Lung Metastasis on 68Ga-Labeled Prostate-Specific Membrane Antigen PET/CT in a Patient With Prostate Cancer. Clin Nucl Med 2017;42:485-6.
Cite this article as: Wu LX, Lei L, Zhu YC, Du KQ, Li XF, Chen HF, Wang WX, Xu CW. A prostate cancer patient with isolated lung metastases: a case report. Transl Cancer Res 2020;9(3):2064-2068. doi: 10.21037/tcr.2020.01.19 